\title{
Barreras para el Aprendizaje y la Participación en la Escuela del Alumnado con Dislexia: Voces de Familias
}

\author{
Barriers to Learning and Participation in School of Students \\ with Dyslexia: Family Voices
}

\author{
Dolors Forteza * \\ Laura Fuster \\ Francisca Moreno-Tallón
}

Universitat de les Illes Balears, España

\begin{abstract}
Alcanzar una educación de calidad, nos remite a la educación que debe ser inclusiva y equitativa, poniendo énfasis en el valor de las diferencias para mejorar la enseñanza y las experiencias de aprendizaje. Pero, todavía son muchos los que sufren procesos de exclusión en la escuela; los que tienen un diagnóstico de dislexia conforman un grupo dañado por prácticas y culturas de centro escasamente acogedoras. El presente estudio se orienta a analizar las barreras que frenan su aprendizaje y las secuelas que producen. Desde un enfoque metodológico de corte biográfico-narrativo, las voces de familias, a través de la entrevista, revelan que son múltiples los obstáculos que, más allá de problematizar el progreso de sus hijos e hijas, perjudican su autoconcepto y autoestima. Los resultados muestran como principales barreras aquellas que están relacionadas con la falta de comunicación eficiente escuela-familia, metodologías de aula que por su naturaleza magnifican las dificultades en la lectura y la escritura repercutiendo en el aprendizaje, y el impacto emocional producido por la ausencia de respuestas a las necesidades del alumnado con dislexia. Visibilizar estas barreras es un compromiso con el derecho a una educación de calidad para y con todos en la educación obligatoria.
\end{abstract}

Descriptores: Educación inclusiva; Barreras; Dislexia; Familia; Igualdad de derechos.

Achieving quality education refers us to education that must be inclusive and equitable, emphasizing the value of differences in order to improve teaching and learning experiences. But, there are still many who suffer processes of exclusion in school; those who have dyslexia make up a group damaged by poorly welcoming practices and center cultures. The present study is oriented to analyze the barriers that hinder their learning and the sequels that produce. From a biographicalnarrative methodological approach, the voices of families, through the interview, reveal that there are multiple obstacles that, beyond problematizing the progress of their sons and daughters, harm their self-concept and self-esteem. The results show as main barriers those related to the lack of efficient school-family communication, classroom methodologies that by their nature magnify the difficulties in reading and writing impacting on learning, and the emotional impact produced by the absence of responses to the needs of students with dyslexia. Making these barriers visible is a commitment to the right to quality education for and with all in compulsory education.

Keywords: Inclusive education; Barriers; Dyslexia; Family; Equal rights.

*Contacto: dolorsforteza@uib.es

ISSN: 2254-3139

www.rinace.net/riejs/

revistas.uam.es/riejs
Recibido: $\quad 5$ de octubre 2019

$1^{\text {a }}$ Evaluación: 16 de noviembre 2019

$2^{\text {a }}$ Evaluación: 29 de noviembre 2019

Aceptado: $\quad 5$ de diciembre 2019 


\section{Introducción}

Caminar hacia la inclusión no es una tarea fácil, al contrario, dada su complejidad es imperativo ponerse en movimiento. Esto es, sacudir inercias, prejuicios, actitudes; reflexionar sobre el currículum, las metodologías, la evaluación, los materiales; y analizar el proyecto de escuela, el por qué y el para qué, entre otros muchos factores que impregnan la dualidad 'exclusión-inclusión'. Es ineludible escudriñar cómo los contextos escolares limitan las oportunidades de aprendizaje negando el desarrollo óptimo de las singularidades propias de la diversidad humana. En el siglo XXI es inexcusable profundizar en "cómo" el derecho a una educación de calidad se ejerce de manera desigual; una barrera potente que actúa sobre los grupos más vulnerables. La alternativa a la exclusión y la discriminación es, sin duda, una educación que incluye, que aprecia la diversidad como un valor, y mengua la categorización a la que el sistema educativo está tan acostumbrado.

El concepto de "barreras al aprendizaje y la participación" de Booth y Ainscow (2015, p. 9 ), esencia de sus planteamientos educativos desde hace unas décadas, sigue siendo un eje central para comprender las desventajas y desigualdades que se van conformando en el sistema educativo, en muchos casos, al limitar la participación y las posibilidades de aprender, consiguiendo, así, la despersonalización de la enseñanza a la que aluden Echeita y Domínguez (2011): "hacer todos lo mismo, al mismo tiempo, con los mismos medios o apelando a idénticas formas de motivación” (p. 29).

Slee (2012) advierte que la educación inclusiva "no es un problema técnico que resolver mediante un conjunto de medidas compensatorias, sean adaptaciones curriculares, la adaptación física de la escuela, la forma de la prueba [...]. Estos enfoques no llegan a cuestionar la arquitectura de la exclusión" (p. 161). O, dicho de otra manera: "el papel de la escuela respecto al mantenimiento o reducción de las desigualdades depende de lo que haga la escuela, no es una situación exclusivamente estructural" (Murillo y HernándezCastilla, 2014, p. 17). Al compartir estos posicionamientos consideramos relevante seguir ahondando en el análisis de barreras que, de formas variadas, imposibilitan o dificultan el aprendizaje de niños, niñas y jóvenes creando desigualdades. Sobre esta base se ha planteado el estudio, como responsabilidad, de acuerdo con López (2012), en la búsqueda de "un nuevo proyecto educativo que nos permita aprender a convivir como una oportunidad para la libertad y la equidad” (p. 131).

Reconocer esas barreras es una forma de hacer visible la fragilidad del grupo de alumnado con un diagnóstico de dislexia en un sistema educativo que no acoge y no valora la diversidad, incidiendo en su rendimiento y su estado emocional. Un sistema educativo que "nunca puede ser de calidad si mantiene en su seno mecanismos de naturaleza excluyente" (Azorín y Sandoval, 2019, p. 24).

\section{Revisión de la literatura}

El objetivo de este estudio es ahondar en el análisis de las barreras a las que deben enfrentarse los alumnos con dislexia en el ámbito escolar desde la perspectiva de las familias; barreras que obstaculizan el aprendizaje de sus hijos e hijas y que devienen en trayectorias escolares y familiares insatisfactorias. La revisión de la literatura nos permite contrastar las evidencias científicas con las experiencias que viven las familias, profundizando en la comprensión de esas barreras. 


\subsection{Aproximación a la dislexia}

La International Dyslexia Association (AID, 2002) la define como una dificultad específica de aprendizaje de origen neurológico, caracterizada por dificultades de precisión y fluidez en el reconocimiento de palabras escritas y por problemas en la ortografía, la descodificación y el deletreo. Su prevalencia en las escuelas se ha estimado en un 5\%-15\% de alumnos (Soriano-Ferrer y Piedra, 2014) y es por ello que este grupo heterogéneo presenta cada vez más una presencia relevante en nuestras aulas.

Según el DSM-5 (American Psychiatric Association, 2014), el diagnóstico diferencial de la dislexia se incluye dentro de los trastornos del neurodesarrollo, como un trastorno del aprendizaje con dificultades en la lectura y en la expresión escrita. Investigaciones actuales se centran en el desarrollo de las representaciones fonológicas (Cuetos, Soriano y Rello, 2019) y cómo estos componentes se relacionan con el aprendizaje de la lectura y la escritura y, por consiguiente, con la dislexia (Cuetos, Suárez-Coalla, Molina y Llenderrozas (2015). El alumnado con un diagnóstico de dislexia puede experimentar dificultades de comprensión en la lectura y en la ortografía (Defior, Serrano y GutiérrezPalma. 2015), que le supondrán momentos de baja autoestima y problemas emotivosconductuales (Zuppardo, Serrano y Pirrone, 2017).

Por todo ello, se hace necesaria una adecuada detección de estas dificultades, para sortear el fracaso escolar e impedir el sufrimiento del niño y la familia (Cuetos et al., 2015), ya que las investigaciones muestran que una intervención temprana es mucho más efectiva en torno a los $4-4,5$ años, debido a la plasticidad del cerebro que caracteriza los primeros años de vida (Cuetos et al., 2015; Hatcher, Hulme y Snowling, 2004; Papanicolaou et al., 2003).

\subsection{Dislexia y educación}

Ante la prevalencia y las necesidades que presentan los alumnos con dislexia en las aulas, es primordial que los docentes profundicen en el aprendizaje de la lectura. Una investigación de interés en este sentido es la de Echegaray y Soriano (2016) llevada a cabo en la Comunidad Valenciana con maestros en ejercicio y en fase de formación. Concluyen que los maestros con y sin experiencia desconocen los problemas emocionales y/o sociales de los alumnos con dislexia, las alteraciones neurofuncionales y neuroanatómicas y los problemas de lateralidad, y los maestros en formación inicial tienen, además, la creencia de que la dislexia puede curarse. Algunos de sus resultados coinciden con el estudio realizado por Binks y otros (2012) en torno al conocimiento de los maestros sobre la lectura y ultiman que no poseen conocimientos sólidos acerca de los diferentes aspectos que conforman el lenguaje (fonética, morfología, sintaxis y fonología), base de la enseñanza del proceso lector, lo que repercute, especialmente, en alumnos con dislexia.

Otras investigaciones empíricas han probado la eficacia de la enseñanza multisensorial en el desarrollo de habilidades de lectura. Jeyasekaran (2015) examinó la efectividad de utilizar todos los canales sensoriales con niños con dislexia en la India. Sus hallazgos indican una diferencia estadística significativa antes y después de la intervención, con una mejora de la lectura del $12 \%$.

En la misma dirección que la anterior se dirige la investigación de Soliman y Al-Madani (2017). El objetivo principal es examinar, en un clima emocional seguro, los efectos de la instrucción multisensorial en la lectura, fluidez y comprensión de niños árabes de $4^{\circ}$ grado con dislexia. Los resultados revelan que hubo diferencias estadísticamente significativas 
en las pruebas posteriores de fluidez (que incluyen precisión y velocidad de lectura) y de comprensión lectora entre el grupo control y el experimental; en este último se evidencia la exitosa intervención a través de actividades que ponen en juego los diferentes sentidos.

Las TIC son un elemento adaptable, personalizable y motivador para el alumnado, y favorecen la metodología multisensorial. Para Gasparini y Culén (2012) su uso en las aulas anima, al alumnado con dislexia, a la lectura y minimiza el estigma que producen sus dificultades; esta es la conclusión más relevante que extraen al realizar un estudio de casos comparando la memoria y la comprensión al trabajar con tabletas o en papel; los resultados muestran mejores puntuaciones en las pruebas de lectura al utilizar herramientas tecnológicas.

Anestis (2015) diseñó dos pruebas no estandarizadas, una en formato digital y la otra en papel incluyendo operaciones matemáticas básicas. Los estudiantes con dislexia obtuvieron un $18 \%$ más de respuestas correctas en la prueba realizada con ordenador; en cambio el grupo control no tuvo diferenciaciones importantes en sus actuaciones; por otra parte, concluye que el uso de las TIC en el procedimiento de evaluación puede contribuir a una mejor concentración.

\subsection{Familia y estado emocional}

Robledo y García (2014) llevaron a cabo un estudio comparativo para evaluar el clima familiar en tres grupos de niños: con dislexia, TDAH y de rendimiento escolar normal (RN). Los resultados revelan que en los dos primeros grupos existe más tensión. Se deduce de esto que las familias con un hijo con dislexia o TDAH tienen una preocupación persistente respecto a las necesidades de sus hijos, dedicando el mayor tiempo de que dispone el niño, fuera de las horas lectivas, a la realización de tareas académicas, asistir a terapias específicas, para paliar las consecuencias de un proceso escolar inadecuado o insuficiente. Concluyen, asimismo, que los padres e hijos pertenecientes a grupos RN son mucho más optimistas en comparación a los otros dos. Los grupos de alumnos con dislexia y TDAH perciben bajas expectativas por parte de sus familias y maestros, que les conduce a tener una opinión desfavorable sobre su propio desempeño académico.

Alexander-Passe (2007) investiga el nivel de estrés de los niños con dislexia en la escuela. Los resultados sugieren diferencias entre los grupos, sin y con dislexia. Estos últimos experimentan un elevado estrés, que se produce en las interacciones con maestros, respecto a los exámenes y el rendimiento académico; por consiguiente, se generan emociones (miedo, timidez y soledad) y manifestaciones fisiológicas (náuseas, temblores o latidos rápidos del corazón) que hieren su autoconcepto. El estudio también evidencia que se plantean la impresión que causan en sus compañeros y no gozan de gran autoestima.

Bryan, Burstein y Bryan (2001), a través del análisis de un conjunto de investigaciones, explican la realidad que viven las familias respecto a los deberes de sus hijos con dislexia. Estas tareas suelen estar estructuradas de forma que requieren de las habilidades en las que tienen mayores dificultades como son la compresión, escritura, decodificación..., provocando un menor rendimiento. En este sentido, la ayuda de un familiar se convierte en un elemento básico; ayuda que, por la frustración que acarrea, acaba generando malestar y discordia alrededor de las tareas escolares, desmotivación de los hijos, y dudas de los progenitores sobre su autoeficacia para ayudarles.

En la misma línea, Zuppardo, Serrano y Pirrone (2017), en su estudio con una muestra de 25 alumnos diagnosticados de dislexia y disortografía y 10 sin dificultades en la lectura y 
la escritura, definen un perfil emotivo-conductual de los niños con dislexia que muestran una baja autoestima y malestar psicológico comportamental (ansiedad) ocasionados, principalmente, por las propuestas didácticas tradicionales de la escuela.

\section{Método}

La posición que asumimos en este estudio se inscribe en el amplio abanico de posibilidades que ofrece el enfoque cualitativo de investigación. El método biográfico-narrativo es el que nos permite adentrarnos, como apunta Van Manen (2003), en las experiencias significativas de la vida diaria, las de familias a cuyos hijos se les ha diagnóstico dislexia. Este enfoque es el que nos permite analizar las relaciones entre situaciones específicas y sus contextos (Álvarez y San Fabián, 2012) y, posteriormente, transformar la palabra oral (las voces) en palabra escrita, captando los significados de esas experiencias singulares. $\mathrm{Al}$ final, cimentamos un relato compuesto de micro-relatos de acuerdo con los requerimientos de la investigación en educación y los componentes éticos de la misma; una construcción particular que surge del contraste entre las investigadoras y de estas con la revisión de la literatura.

Es conveniente matizar que las investigadoras coinciden en un interés común, fruto de nuestras experiencias: en la escuela, en los centros de educación secundaria, en la universidad, con las familias, y con otros investigadores en la temática tanto de la dislexia como de la educación inclusiva. Nuestros ámbitos de trabajo interseccionan en el tiempo y confluyen en un diálogo que se enriquece de las propias aportaciones experienciales.

La herramienta básica para la recogida de información es la entrevista semiestructurada como medio para "reunir material narrativo experiencial que en un momento dado puede servir como recurso para desarrollar un conocimiento más rico y más profundo sobre un fenómeno humano" (Van Manen, 2003, p. 84). Siguiendo las recomendaciones del mismo autor, decidimos que fuera semiestructurada para no dejarnos llevar "por las entrevistas que van a todas partes y a la vez a ninguna" (p. 84), con el objetivo de responder a una pregunta clave: ¿qué vivencia tienen las familias en relación a las trayectorias escolares de sus hijos e hijas con un diagnóstico de dislexia?

\section{Procedimiento}

Las personas participantes han sido progenitores (madres o padres) con hijos e hijas con dislexia que han tenido o tienen relación con la asociación Dislexia y Familia (DISFAM). De un listado inicial de 10 familias que por determinadas circunstancias se estimaron más accesibles, se consiguió contactar, por vía telefónica, con 6 que accedieron a participar. En el primer contacto telefónico se expusieron las siguientes cuestiones: presentación de quien hablaba, cuál era el propósito de la llamada y la finalidad del estudio. Una vez aceptada la colaboración voluntaria, se estableció una fecha, hora y lugar para realizar la entrevista, considerando las necesidades específicas de los participantes.

Antes de empezar se presentó a los entrevistados el documento de consentimiento informado. Se leyó en voz alta y se firmó por duplicado. Durante las entrevistas, se mantuvo, en todo momento, una interacción respetuosa, creando un clima de empatía y confianza, además de mantener firmemente los criterios éticos que deben guiar cualquier investigación en educación. Todas las entrevistas fueron registradas en audio y transcritas en su totalidad. 
Las preguntas formuladas en la entrevista, que emergen de la información extraída de la revisión de la literatura, giran en torno a tres fases tal y como se especifica en el cuadro siguiente.

Cuadro 1. Contenido de la entrevista y fases en las que se desarrolla

\begin{tabular}{|c|c|}
\hline BLOQUES & CONTENIDO DE LAS PREGUNTAS \\
\hline $\begin{array}{l}\text { 1. Fase inicial de } \\
\text { contextualización }\end{array}$ & $\begin{array}{l}\text { Número de hijos. } \\
\text { Edades. } \\
\text { Nivel que están cursando. } \\
\text { Repetición de algún curso. } \\
\text { Edad en la que se diagnostica la dislexia. } \\
\text { Quién realiza el diagnóstico. }\end{array}$ \\
\hline $\begin{array}{l}\text { 2. Fase intermedia de } \\
\text { preguntas con carga } \\
\text { emocional }\end{array}$ & $\begin{array}{l}\text { Momento y sospechas que hacen pensar en una dificultad. } \\
\text { Cómo fue este proceso, fueron los maestros que observaron las } \\
\text { dificultades y contactan con las familias o fueron estas que } \\
\text { acuden a la escuela para contrastar información. } \\
\text { Recorrido de las familias desde que temen una dificultad hasta } \\
\text { que se detecta la dislexia. } \\
\text { Cómo surge la decisión de hacer el diagnóstico, quién lo } \\
\text { sugiere. } \\
\text { Qué sentimiento produce el resultado del diagnóstico en la } \\
\text { familia y los hijos. } \\
\text { Cuál es la reacción de los maestros frente al diagnóstico. } \\
\text { Qué ocurre en la escuela después del diagnóstico. } \\
\text { Cómo se afrontan los exámenes y deberes. } \\
\text { Qué apoyos, materiales y humanos, se reciben por parte de la } \\
\text { escuela. } \\
\text { Qué dificultades encuentran las familias actualmente en la } \\
\text { escuela. }\end{array}$ \\
\hline 3. Fase final de síntesis & $\begin{array}{l}\text { Visión sobre el futuro académico de los hijos con dislexia. } \\
\text { Información que se considere relevante añadir. }\end{array}$ \\
\hline
\end{tabular}

Fuente: Elaboración propia.

De la selección y reducción de la información aportada por los participantes emergen las categorías de análisis. Los pasos que involucran este procedimiento requieren un proceso de enfoque sobre las unidades temáticas que son notables y acordes con el objetivo de la investigación. La codificación de estas unidades es la primera fase que realizamos para seleccionar la información a través de reiteradas lecturas de las entrevistas. Progresivamente, aglutinamos la información en categorías (fase de reducción) sobre las que pivotará el análisis de los resultados. Para ello elaboramos una matriz de doble entrada, en la que situamos en cada categoría información literal ya codificada de los participantes. Cuatro son las categorías globales resultantes de este proceso: el diagnóstico, las actitudes de los docentes, la metodología en el aula, el estado emocional de las familias y sus hijos.

Los participantes del estudio han sido:

- Familia 1: formada por madre y padre con dos hijos de edades de siete y diez años. Actualmente, la hija mayor está realizando sus estudios de primaria en un centro público; se sospecha que pueda tener altas capacidades además de la dislexia.

- Familia 2: cuyo núcleo familiar está constituido por cuatro miembros, padre y madre con una hija de trece años que está realizando segundo de la ESO en un instituto. Realizó la educación primaria en un centro público, el mismo en el que 
está su hermano de siete años cursando segundo de primaria, con diagnóstico de dislexia y TDAH.

- Familia 3: familia numerosa formada por padre y madre con cuatro hijos con dislexia de edades de veintiocho, veinte, diecisiete y doce. La hija de 12 años está escolarizada en un centro privado que se rige por el sistema educativo inglés.

- Familia 4: constituida por padre y madre, actualmente separados. La hija mayor, de 17 años, está en un instituto haciendo bachiller y el pequeño, de 12, no se ubica en un nivel concreto ya que asiste a una escuela activa; anteriormente, fue a una escuela cooperativa, la misma que su hermana.

- Familia 5: formada por padre y madre con tres hijos de edades de 27, 13 y 11 años. Actualmente el mayor está estudiando fuera, la segunda hija está en la ESO y el tercer hijo está en sexto de primaria, quien tiene un diagnóstico de dislexia.

- Familia 6: familia en la que el padre y la madre están separados. Tienen tres hijos, dos con 9 años y uno de 20 con dislexia y discalculia. Ha pasado por tres centros públicos y actualmente está acabando una formación básica.

\section{Resultados}

Organizamos los resultados en torno a tres dimensiones: desencuentros, prácticas en el aula y efectos emocionales. Las dos primeras se relacionan con las barreras mientras que la última incide en los impactos que estas barreras producen en el ámbito familiar (progenitores e hijos). En cada uno de estos apartados se aglutinan evidencias de la experiencia de los participantes; su voz da sentido a esta estructura.

Para identificar a las familias se sigue el orden de presentación que se ha realizado con anterioridad, identificando el progenitor (M: madre o P: padre) que participó en la entrevista: F1M, F2M, F3M, F4M, F5P y F6P.

\subsection{Desencuentros familia-escuela}

Uno de los primeros desencuentros a los que se refieren las familias hace alusión a la reiterada negación de los docentes a aceptar el diagnóstico de dislexia que proviene, por lo general, de profesionales externos a los centros. Negativa que en algunos casos se traduce en manifestar buenas intenciones de cambio en el aula sin efectos reales.

La escuela responde que van a hacer todo lo que les hemos comentado... "Tranquila, sabemos qué hacer". Pero en la realidad no se ha hecho nada. [...] El tutor se negó desde el principio a aceptar la dificultad de nuestra hija y expresó 'no llega, no es igual que sus amigos'. Aceptó que no iba a hacer absolutamente nada con su dislexia. (F2M)

No te preocupes, estamos acostumbrados a dislexias, estamos formados, esto nos decían, el cuento de siempre... (F3M)

El maestro dice que está abierto, pero no es eso lo que nos ha de decir, sino que está abierto con nuestro hijo; es él que ha de decir cómo lo ve y qué cosas cree que podemos aportar nosotros como padres. (F5P)

La tutora dice que nuestra hija no tiene ninguna dificultad, negando el propio diagnóstico. (F1M) 
Comentarios como los siguientes refuerzan la idea anterior; se recalca el desconocimiento que tienen los tutores en la escuela sobre la dislexia y cómo esta afecta a su aprendizaje, reflejándose en expresiones de los maestros con falta de sensibilidad. Así lo manifiestan.

Al niño hay que empujarle más... (F4M)

Le preguntamos al profesor por qué había suspendido catalán y nos dio una respuesta muy a la antigua: hará que se espabile. (F5P)

Que vuestro hijo estudie más y se esfuerce más, ha de poner codos y lo sacará; esto es lo que nos decían, pero los medios eran los mismos para él que para el resto de su clase $[\cdots] \cdot(\mathrm{F} 6 \mathrm{P})$

Es significativa la actitud negativa que pone de manifiesto un progenitor para coordinarse la escuela con un profesional externo que trabaja con su hijo fuera del horario escolar.

Les hubiera podido ir bien que les explicara cómo ayudar a nuestro hijo, pero los maestros tienen la potestad de decir sí o no, marcan las pautas, y no han querido coordinarse a propuesta de esta profesional. (F5P)

Una actitud adversa también se refleja en los procesos de comunicación entre los docentes. Los padres deben asumir el rol de 'puente', entre los maestros de distintos cursos o del mismo nivel.

Es complicado, me refiero a la coordinación. Al siguiente año tienes que volver a explicar todo cuando hay cambio de maestro, y parece que estás vendiendo tu moto. $(\mathrm{F} 1 \mathrm{M})$

La coordinación entre ellos no funciona, es un esfuerzo que ha de hacer la familia, esto no es natural. (F5P)

Una madre narra un episodio vivido con una profesora, con una actitud amenazante y falta de comunicación con sus compañeros; tiene que ver con el suspenso de una asignatura durante dos trimestres seguidos.

Es que tu hijo no sabe inglés. Me quedé mirándola y le digo: ¿no has mirado sus papeles?, de dónde viene, lo que tiene... y dice: "aquí nos pasamos toda la información..." No había leído absolutamente nada. $Y$ había pasado el segundo trimestre y dijo lo mismo. Yo pensé: de aquí nos vamos. (F3M)

Destacar, por último, un desencuentro provocado por las manifestaciones de los maestros respecto a las posibilidades de aprendizaje. Son, en palabras de las familias, nocivas, destruyen progresivamente la autoestima, como si no fuera suficiente con el comportamiento global de negación de las dificultades que caracteriza las experiencias escolares.

Una maestra le dijo a mi hija por algo que no hizo bien que la había decepcionado mucho. (F1M)

Le dijeron en la escuela 'no vas a poder, ¿̇por qué lo vas a hacer? es que no hace falta ni que lo intentes... es que no vas a poder'. (F3M)

\subsection{Las prácticas en el aula: Dime qué haces y te diré cómo enseñas}

Las TIC utilizadas como recurso pueden facilitar el aprendizaje del alumnado con dislexia, Sin embargo, hay docentes que consideran que su uso provoca una condición de desigualdad con respecto a sus compañeros, tal y como expresa la siguiente familia.

To decía: "puede llevar grabadora” NO.. y ¿̇un ordenador? No, porque está aprendiendo a escribir. NO, todo era NO porque esto es hacer diferencias y porque hay aquí otros alumnos con dislexia y no utilizan este tipo de cosas. $T$ a no solo es que no nos dejaran un ordenador, es que no nos dejaban llevarlo de casa. (F4M) 
Esta restricción es un sinsentido teniendo en cuenta que las herramientas tecnológicas son útiles y un recurso necesario para la realización de los deberes y para el estudio en casa; recursos que, por otro lado, forman parte de su vida cotidiana.

Ella lee, lo graba y luego se escucha. Ella busca sus medios para llegar... (F2M)

La medida de 'repetición' está a la orden del día en los centros. Una solución cuyas consecuencias recaen en el alumno, sin cuestionarse la responsabilidad de los docentes en esta medida. Todas las familias expresan desacuerdo con esta respuesta ya que, si los maestros no han trabajado a lo largo del curso teniendo en cuenta las necesidades, no tiene significado repetir.

Persistí mucho en que mi hija en primaria no repitiera, con mucha pelea conseguí que la fueran aprobando hasta segundo... "mientras vosotros no estáis haciendo todo lo que ella realmente necesita va a seguir aprobando"; para repetir necesitaban mi firma. $(\mathrm{F} 2 \mathrm{M})$

La metodología utilizada en los centros de primaria o secundaria donde han ido o se encuentran los alumnos de las familias entrevistadas, mayoritariamente es tradicional fundamentada en el libro de texto.

Sí, utilizaban libros. En los colegios no había realmente un sistema sin libros ni con proyectos ni nada, era un sistema totalmente tradicional. (F3M)

Una de las madres (F1) explica que la metodología utilizada varía según la asignatura. Mientras que las hay que trabajan por proyectos, en las áreas de lengua se trabaja con el libro de texto como vertebrador de la clase.

Las familias demandan adaptaciones ante las experiencias tradicionales que son iguales para todos. Adaptaciones que, al fin y al cabo, no suponen un sobre esfuerzo al profesorado.

La niña era un fracaso total ¿̨pero por qué era un fracaso total? porque no se hacía ninguna adaptación. (F2M)

Tienen derecho a una adaptación y además yo exijo que se les haga esa adaptación, ¿por qué? porque tienen derecho, punto. Son mis hijos y quiero que se cumplan sus derechos, es que no hay otra... (F3M)

En algunos casos los niños se sacan del aula para recibir apoyo a pesar de que las familias no están de acuerdo.

Sale de la clase. Pero pierde contenidos, información... (F2M)

Las familias son las primeras en expresar que sus hijos necesitan otra forma de aprender, ya que observan que la ofrecida por el centro no es la más adecuada para ellos. La repetición de contenidos, como aprendí yo a él no le va bien. Todo lo que sea
acústico y visual le funciona. $(\mathrm{F} 6 \mathrm{P})$

Los deberes son otro caballo batalla. Estas tareas provocan, tal y como indican, que sus hijos deban dedicar muchas horas para cumplir con esta obligación, sin dejar tiempo para otras actividades.

Los deberes es una cosa exagerada en primaria, la niña comía y se sentaba a hacerlos y eran las once de la noche y seguía... le tuve que quitar de natación y de baile porque no le daba tiempo. (F2M)

Está dos horas por un trocito cuando los demás están... la diferencia de tiempo es muy evidente. Los demás hacen en 10 minutos lo que ella tarda una hora. (F3M) 
Las familias son muy críticas con los deberes porque les generan mucha tensión en casa por el tiempo excesivo que, a menudo, han de dedicarle, tanto sus hijos como los propios progenitores.

Para mí hacer los deberes ahora mismo con mi hijo me lleva toda la tarde para hacer una hoja. (F2M)

Mi hija cuando está haciendo los deberes se pone nerviosa, se frustra. (F3M)

Fueron gritos, fue tensión en casa. No entendía por qué un día mi hija conseguía escribir una palabra bien y otro día no lo conseguía. ¿̇ cómo es que ayer sabía escribir su nombre y hoy no sabe? ¡Teníamos unas peleas en casa! porque le reñia un montón. Claro, esto fue antes de tener el diagnóstico de dislexia. (F2M)

Se comenta también el tipo de deberes de fin de semana o de vacaciones, actividades muy tradicionales y mecánicas, que requieren alta dedicación de tiempo.

Los deberes de final de semana son prehistóricos... tienes que leer ese libro, pero pasa que el libro tiene tanto contenido que no puede, y yo me pongo a su lado y va leyendo, y le pregunto sobre los personajes del libro, qué parte le ha gustado más... Cada semana lo mismo, llevamos 2 años haciéndolo. (F2M)

\subsection{Efecto emocional en las familias}

Los resultados dejan entrever el estado de ánimo de padres e hijos. Reflejan angustia por lo que han vivido y siguen viviendo con las barreras que encuentran en la escuela que están afectando al estado emocional de sus hijos.

La escuela no entiende el mal que hace, porque si fueran conscientes no lo harian. $(\mathrm{F} 4 \mathrm{M})$

No hay derecho que los pataleen de esta manera. (F3M)

Cuando se confirma el diagnóstico es un momento delicado para las familias. Narran, así, su experiencia de desazón y tristeza.

Deja a la familia muy débil. (F1M)

Me afectó más el diagnóstico de mi hija que de mi hijo; no paraba de llorar porque no quería que ella sufriera. Con mi hijo ya me lo tomé de otra manera. (F2M)

Lo pasamos todos muy mal la verdad, fatal, al ver, además, el decaimiento de tus hijos, con la presión en esas edades... (F3M)

Después del dictamen, las familias esperan un cambio por parte de los docentes y, sin embargo, continúan con sus prácticas habituales. Esto provoca desesperación y tirantez que, en ocasiones, lleva a la confrontación familia-escuela.

Nos dijeron que éramos malos padres, te hacen sentir como malos padres, y te dicen que la niña está sufriendo mucho, que el problema de la niña es que no llega porque ve que todos los amigos ya leen y ella estaba empezando. (F2M)

Empezó la lucha entre la escuela y yo. Entiendo que ellos pensaran que era pesada, que tenía a mi hijo muy protegido, que el niño es un mimado... Lo que pasa es que llega un momento que tanto enfrentamiento y tanto cansancio, y todo... ya no puedes más. (F4M)

La verdad que al final de este curso yo ya he tirado la toalla, ya no puedo más, prefiero dedicarme más a mi hijo que intentar hacer un cambio en la escuela. [...] nosotros hemos tomado la decisión de mantener cierta marginalidad, tenemos una relación formal adecuada, hemos tomado la posición de no confrontación, de acabar bien la primaria. (F5P)

Las barreras durante el período de escolarización de sus hijos reavivan su preocupación por el presente y por su futuro académico. Tienen claro que su porvenir dependerá mucho 
de los docentes que se encuentren en sus trayectorias, y esta incertidumbre causa una mayor angustia en las familias.

Tú no puedes estar pendiente del maestro que le tocará cada año a tu hijo, cada curso es pensar ¿̇y el año que viene quién va a ser? Porque como sea este o este otro estamos perdidos. $(\mathrm{F} 4 \mathrm{M})$

Las familias afrontan con mucha inquietud las vivencias de sus hijos en la escuela, en la que deben enfrentarse a continuos obstáculos que van minando su estado de ánimo y su salud.

Empezamos a ver que estaba realmente hundido y con un principio de depresión... decía que nada, pero llevaba la mochila puesta detrás y entraba a la escuela arrastrando los pies con una desgana, con una pena, con una cosa ... que a mí me dolía dejarlo ahí. (F3M)

Mi hijo no quería ir a la escuela. Dónde se ha visto que un niño no quiera ir a la escuela con sus compañeros, a aprender... es muy fuerte. Mi hijo, un niño que dejó de bailar, siempre se escondía, siempre se escondía debajo de las mesas [...], iba a la escuela y me decían que el niño se portaba fatal, cada día lo sacaban al pasillo. $Y$ yo decía: "no entendéis nada". (F4M)

\section{Discusión y conclusiones}

Los maestros de nuestro sistema educativo presentan una falta de conocimiento sobre la dislexia, tal y como ya se planteado en la investigación de Echegaray y Soriano (2016). Esta falta de saber se ha visto reflejada en este estudio a través de la idea generalizada de la repetición como solución a las dificultades sin cuestionar las prácticas en el aula. Se niega, en ocasiones, la existencia de la dislexia, tratándose como meras dificultades que puede presentar cualquier niño en su recorrido escolar. La solución no es repetir curso; el remedio pasa por una transformación profunda del sistema educativo que debería estar obligado a acoger a todos los niños independientemente de sus diferencias particulares.

La carencia de información-formación por parte de los docentes dificulta una atención adecuada a las necesidades de este grupo de alumnos para conseguir, en la acción cotidiana del aula, un aprendizaje exitoso (Bueno, 2017). Para Blanco (2009) las dificultades de estos alumnos les acompañaran toda la vida y el hecho de repetir un currículo no cambiará la manera de procesar la información, necesitan herramientas específicas de aprendizaje y otras maneras de acceder a la información, como por ejemplo las TIC, ya que posibilitan la personalización a las necesidades del alumnado.

Esta falta de conocimiento se ve doblemente afectada por la actitud de los docentes que enfocan su mirada erróneamente hacia las dificultades de los alumnos, a menudo invisibilizándolas y, en consecuencia, frustrando los esfuerzos que hacen para aprender. Una actitud que se refuerza por las prácticas que desarrollan en sus aulas, dirigidas a un alumno ideal, con una metodología tradicional que solo favorece a una parte del alumnado dejando fuera al resto, siendo el colectivo de alumnos con dislexia desfavorecidos por unas culturas, políticas y prácticas que se fijan "meramente en sus fracasos y no en cómo satisfacer sus necesidades" (Forteza y Moreno, 2017, p. 42).

Es fundamental afrontar la enseñanza con una actitud abierta al cambio, a la indagación de nuevas maneras de hacer en la escuela, confiando en las posibilidades de aprendizaje de todos y cada uno de los alumnos, y más, si cabe, de aquellos que pueden ser más vulnerables a procesos de exclusión, debido a sus dificultades de partida a las que no se 
responde de manera adecuada en los centros. Contrariamente, una actitud que debilita, es una potente barrera porque limita el aprendizaje de los alumnos con dislexia, y también de todos; una actitud que va generando rechazo hacia la escuela, como ya hemos visto, y va calando en la autoestima y autoconcepto de los niños con dislexia ya en la educación primaria llegando a interiorizar que son 'tontos', 'vagos', 'incapaces de aprender y estudiar'.

Un sistema trasnochado que se centra casi exclusivamente en el libro de texto dificulta, a los alumnos con dislexia, las oportunidades de acceso a los aprendizajes (Asensio, 2016). La diversidad del alumnado en el aula requiere metodologías que integren el uso de diversos canales sensoriales para aprender (Jeyasekaran, 2015; Soliman y Al-Madani, 2017), y la utilización de recursos variados, como las TIC, que favorecen el proceso de enseñanza y aprendizaje (Anestis, 2015).

Los deberes, por su parte, engendran insatisfacción en las familias porque restringen sus necesidades de ocio y tiempo libre. Porque se basan en tareas de lectura y escritura, justo donde residen las dificultades. Porque crean dependencia del adulto, y un clima de tensión y frustración en el seno familiar que no hacen más que agravar la situación (Bryan, Burstein y Bryan, 2001).

Por último, queremos remarcar que el diagnóstico actúa como barrera cuando las actitudes desfavorables de los docentes, aferrados a metodologías tradicionales, aumentan las bajas expectativas de éxito sobre el alumnado, centrándose en esperar su fracaso. Diagnóstico que, a menudo, llega mal y tarde ya que las dificultades específicas pasan desapercibidas durante años (Armenteros, 2017); se magnifican, así, las dificultades y los niños consolidan sentimientos negativos hacia ellos mismos. Por otro lado, a la carga económica que supone la realización de un diagnóstico, como manifiestan las familias, se le añade el incremento que implica acudir a instancias externas (maestros de repaso) que minimicen las consecuencias de lo que no se hace en la escuela, o para reflotar a sus hijos con terapias emocionales (Alexander-Passe, 2007; Robledo y Barcía, 2014).

Para finalizar, a modo de conclusión, aportamos una serie de reflexiones que giran sobre cuatro ejes: el aprendizaje, las actitudes, las barreras y el dolor, relacionados con las categorías de análisis establecidas en este estudio.

\section{Sobre el aprendizaje}

Inferimos de todo lo dicho que el desasosiego de las familias se corresponde, en gran parte, con la despersonalización de la enseñanza a la que se hace referencia en la introducción. La homogeneidad de metodologías y recursos para que todos los niños y niñas aprendan lo mismo, en el mismo momento y lugar, se traduce en 'intervenciones terapéuticas' (fuera de la escuela) esperando que el alumnado con dislexia se adapte a un diseño de normalidad preconcebido.

Consideramos que una de las causas de la despersonalización es que los docentes no conocen, o conocen superficialmente, cómo se origina el aprendizaje, menos aún cómo facilitar el de niños, niñas y jóvenes con dislexia. Traemos a colación una frase de Claxton formulada hace más de 30 años: "si los profesores no saben en qué consiste el aprendizaje y cómo se produce, tienen las mismas posibilidades de favorecerlo que de obstaculizarlo" (1987, p. 214). Recogiendo las aportaciones de la literatura anteriormente citadas, destaca la de Binks y otros (2012) al afirmar que los maestros no conocen en profundidad cómo se conforma el lenguaje, lo que repercutirá, en mayor medida, en el alumnado con dislexia. 
Viene a colación lo que expresa Pozo (1999) hace otras tantas décadas: "El tango es cosa de dos. Si los maestros se mueven por un lado y los aprendices por otro, difícil será que el aprendizaje sea eficaz" (p. 336). Así, una consecuencia de ese 'no saber' es la falta de reconocimiento de las biografías personales del alumnado, propiciando experiencias de aprendizaje, quizá valiosas para algunos, pero no para todos. Trayectorias escolares satisfactorias invocan a la aceptación de que la calidad del aprendizaje depende de la competencia del profesorado, admitiendo también que aprender es un acto emocional. Enseñar y aprender son, pues, dos verbos inseparables; el progreso y el éxito del que aprende dependen de la interacción que establece el que enseña con este, de sus competencias como educador y de la confianza en las posibilidades de aprendizaje que todos los alumnos tienen.

A partir de los resultados, se pone en evidencia, de acuerdo con la literatura revisada, que la falta de respuestas o respuestas inadecuadas a las necesidades de los alumnos con dislexia en la escuela ejerce una fuerza negativa sobre las emociones, con repercusión en la motivación, la autoestima y el rendimiento (Zuppardo, Serrano y Pirrone, 2017; Robledo y García, 2014; Alexander-Passe, 2007). Para el bienestar psicológico de todo el alumnado, es muy relevante enfatizar la necesaria creación de ambientes de aprendizaje seguros, acogedores y participativos, en los que cada uno y cada una pueda desarrollar al máximo sus talentos.

\section{Sobre los docentes y sus actitudes}

Reiteramos, acordes con los resultados del estudio y con Ainscow, Booth y Dyson (2006), que la mayor barrera para el progreso de sus hijos son los docentes. Son estos los que acaban siendo constructores de barreras, al negarles el derecho de una educación inclusiva y equitativa. Haciéndonos eco de las palabras de Pujolàs (2015, pp. 20-21):

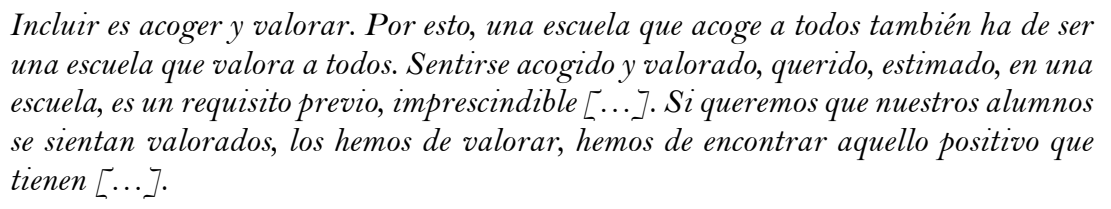
una escuela que valora a todos. Sentirse acogido y valorado, querido, estimado, en una escuela, es un requisito previo, imprescindible [...]. Si queremos que nuestros alumnos se sientan valorados, los hemos de valorar, hemos de encontrar aquello positivo que tienen [...].

Este discurso nos remite a las actitudes que deben acompañar los procesos de enseñanza y aprendizaje de calidad. Valorar a los niños y las niñas y a sus familias implica formas de comunicación que interaccionen con expectativas altas, desplegando para ello estrategias y metodologías que 'tiren' de la capacidad que todos y todas tienen de aprender. En este sentido, lo que manifiesta un padre pone de manifiesto lo que queda por recorrer: "Recuerdo que una PT (siglas de Pedagogía Terapéutica) nos preguntó si nuestro hijo había nacido en domingo, porque era muy vago. Primer diagnóstico que oímos muchas familias". Familias que reclaman a voces una mirada distinta de los docentes hacia sus hijos e hijas.

Pennac (2008) utiliza la metáfora del "fardo" para referirse al cúmulo de condicionantes, frustraciones, miedos... que actúan como una losa que bloquea el crecimiento de los alumnos (a los que se refiere como "zoquetes"). Es significativo que hable del profesorado como factor determinante del éxito o el fracaso escolar; alude a su 'saber ser' y su 'saber hacer':

Nuestros alumnos malos (de los que se dice que no tienen porvenir) nunca van solos a la escuela. Lo que entra en clase es una cebolla: unas capas de pesadumbre, de miedo, de inquietud, de rencor, de cólera, de deseos insatisfechos, de furiosas renuncias acumuladas sobre un fondo de vergonzoso pasado, de presente amenazador, de futuro 
condenado. Miradlos, aquí llegan, con el cuerpo a medio hacer y su familia a cuestas en la mochila. En realidad, la clase solo puede empezar cuando dejan el fardo en el suelo y la cebolla ha sido pelada. Es difícil de explicar, pero a menudo solo basta una mirada, una palabra amable, una frase de adulto confiado, claro y estable, para disolver esos pesares, aliviar esos espíritus, instalarlos en un presente rigurosamente indicativo. (p. 60)

Sobre las barreras para avanzar en la inclusión

Es irrenunciable, como indica Echeita (2017), examinar las múltiples barreras que limitan el aprendizaje y la participación del alumnado. Identificarlas y reducirlas "es una tarea importante de las políticas y la práctica en todos los niveles, para lograr así que ningún estudiante esté en desventaja" (Porter y Towell, 2017, p. 10).

Es un posicionamiento comprometido con los derechos de equidad e igualdad: seguir evidenciando prácticas o situaciones excluyentes (en artículos de investigación, en congresos, en reuniones científicas, en los contactos con las administraciones educativas, en la formación del profesorado de todas las etapas de enseñanza...); y trabajar con las familias, con el fin de "empoderarnos" juntos en la lucha contra las desigualdades, las injusticias, las discriminaciones, desde la creencia de que la educación inclusiva es el camino.

En este sentido, bien podemos decir que compartimos plenamente las palabras de López (2012): "Saber cuáles son las barreras que impiden el aprendizaje y la participación de algunas niñas y de algunos niños en el aula, es, precisamente, el compromiso ético del discurso de la cultura de la diversidad (p.131). Y las de Murillo y Hernández-Castilla (2014) cuando expresan: "los que trabajamos en el mundo educativo tenemos una responsabilidad ética de luchar por un mundo más justo” (p. 29).

El análisis de las barreras realizado en este estudio pone de manifiesto la falta de comunicación entre la escuela y las familias; las fracturas, en este sentido, son un impedimento para el aprendizaje y la participación. Destacamos, al respecto, que es necesario avivar esta conexión considerando "la importancia de construir relaciones sinérgicas entre escuela, familia y comunidad, como uno de los factores clave para avanzar hacia sistemas y escuelas más inclusivas" (Duk y Murillo, 2016, p. 14). Para este fin es fundamental que el equipo docente y directivo adopte una actitud cooperativa con las familias, escuchando sus propuestas y aportaciones y facilitando que se sientan apreciadas y partícipes de la educación escolar de sus hijos (Parody et al., 2019).

Sobre el dolor que produce la escuela

"Para muchos niños, la experiencia de la escuela es la experiencia cotidiana de la humillación y el dolor". Con esta cita de Slee (2012, p. 29), afirmación que recuerda de su período de formación en educación especial, aseveramos que los niños y las niñas con dislexia padecen en la escuela, al igual que su entorno familiar.

"Mi corazón no entiende por qué la infancia, la etapa más dulce de la vida, tenga que ser un mal recuerdo por una dificultad de aprendizaje”. Razón no le falta a este padre que escribió en $2018^{1}$ un texto estremecedor sobre su experiencia con la dislexia severa,

\footnotetext{
${ }^{1}$ Participó en el $9^{\circ}$ Congreso Nacional e Iberoamericano de Dislexia y otras Dificultades Específicas de Aprendizaje “Avanzando en Oportunidades. EduCación con C de Calidad”, Palma de Mallorca, noviembre. Dio su consentimiento para utilizar el texto.
} 
porque su hijo fue 'objeto', que no sujeto, de una escuela que le hundió, que le anuló, llegando al extremo de tal vulnerabilidad que se plantearon si valía la pena que siguiera en ella para sufrir. Es la aparición de una maestra, su tutora en cuarto curso de primaria, la que le salva del agujero oscuro en el que estaba inmerso (como indica su padre "había tocado fondo, y nosotros también").

La esperanza está ahí, en esa maestra o en ese maestro que cree en su profesión, en la educación, en la capacidad de todos los niños y las niñas para aprender... En este estudio hemos visibilizado algunas barreras desde la perspectiva de las familias; las que día a día, junto a sus hijos e hijas, soportan los desencuentros de un sistema educativo que sigue anclado en viejas formas de pensar y hacer. Confiamos en que llegue el momento de poder escribir solo narraciones que hablen del deseo de aprender motivado por maestros que desean enseñar, al reconocer las fortalezas de todos para impulsar nuevos aprendizajes con éxito. Y para que esto suceda "no necesitamos escuelas que hagan actividades y acciones que contribuyan a que la escuela sea más justa. Necesitamos de manera urgente escuelas justas en su plenitud y de manera profunda" (Carneros y Murillo, 2017, p. 146).

Antes de finalizar queremos destacar algunas limitaciones del estudio realizado. Por una parte, la ausencia de triangulación de fuentes de recogida de información; la voz del alumnado con un diagnóstico de dislexia y de maestros en ejercicio completaría el ciclo de contraste para obtener una visión amplia e interconectada de las barreras que frenan o dificultan el aprendizaje y la participación; y, por otra, sería conveniente aumentar el número de participantes para poder relacionar y comparar la información con aspectos contextuales: edad de los hijos e hijas, nivel educativo, tipología de centro (público, concertado, privado)... Es imprescindible, igualmente, considerar como una limitación la exclusiva mirada efectuada sobre las barreras en este trabajo; cabría ampliar el enfoque, de acuerdo con Echeita (2013), incorporando como objetivo de futuras investigaciones el análisis de facilitadores para lograr mejoras en las políticas, culturas y prácticas desde la perspectiva de la inclusión educativa, reforzando, así, el esfuerzo y desarrollo de innovaciones que se están llevando a cabo en muchos centros y aulas que promocionan la presencia, participación y rendimiento de todos los alumnos y las alumnas.

"Cambiar es difícil, pero posible" (Freire, 2001, p. 126).

\section{Referencias}

Ainscow, M., Booth, T. y Dyson, A. (2006). Improving schools, developing inclusion. Londres: Routledge. https://doi.org/10.4324/9780203967157

Alexander-Passe, N. (2007). The sources and manifestations of stress amongst school-aged dyslexics, compared with sibling controls. Dyslexia, 14, 291-313.

https://doi.org/10.1002/dys.351

Álvarez, C. y San Fabián, J. L. (2012). La elección del estudio de caso en investigación educativa. Gazeta de Antropología, 28(1), 1-12.

American Psychiatric Association. (2014). Manual diagnóstico y estadístico de los trastornos mentales. DSM-5. Madrid: Editoral Médica Panamericana.

Anestis, E. (2015). The effects of using information and communication technologies instead of traditional paper based test, during the examination process, on students with dyslexia. Procedia Computer Science, 65, 168-175. https://doi.org/10.1016/j.procs.2015.09.105 
Armenteros, M. D. (2017). Principales dificultades de aprendizaje en las aulas ordinarias de educación primaria de la región de Murcia. Revista Internacional de Apoyo a la Inclusión, Logopedia, Sociedad y Multiculturalidad, 3(1), 73-90.

Asensio, M. J. (2016). El libro de texto: Una barrera para el desarrollo de la escuela inclusiva (Tesis doctoral). Universitat de les Illes Balears. Palma de Mallorca.

International Dyslexia Association (2002). IDA Dyslexia handbook. What every family should know. Baltimore, MD: IDA.

Azorín, C. M. y Sandoval, M. (2019). Apoyos para avanzar hacia una educación más inclusiva en los centros escolares: Análisis de guías para la acción. Siglo Cero, 50(271), 7-27. https://doi.org/10.14201/scero2019503727

Binks, E., Washburn, E., Malatesha, R. y Hougen, M. (2012). Peter effect in the preparation of reading teachers. Scientific Studies of Reading, 16(6), 526-536. https://doi.org/10.1080/10888438.2011.601434

Blanco, A. I. (2009). Dislexia, escuela y exclusión social: Un estudio desde la sociología acerca de la educación especial. Configurações. Revista de Sociologia, 5, 1-25.

Booth, T. y Ainscow, M. (2015). Guía para la educación inclusiva. Desarrollar el aprendizaje y la participación en los centros escolares. Madrid: FUHEM/ OEI.

Bryan, T., Burstein, K. y Bryan, J. (2001). Students with learning disabilities: Homework problems and promising practices. Educational Psychologist, 36(3), 167-180. https://doi.org/10.1207/S15326985EP3603_3

Bueno, D. (2017). Neurociencia para educadores. Barcelona: Octaedro.

Carneros, S. y Murillo, F. J. (2017). Aportaciones de las escuelas alternativas a la justicia social y ambiental: Autoconcepto, autoestima y respeto. REICE. Revista Iberoamericana sobre Calidad, Eficacia y Cambio en Educación, 15(3), 129-150. https://doi.org/10.15366/reice2017.15.3.007

Claxton, G. (1987). Vivir y aprender. Madrid: Alianza Psicología.

Cuetos, F., Soriano, M. y Rello, L. (2019). Dislexia, ni despiste, ni pereza. Todas las claves para entender el trastorno. Madrid: La esfera de los libros. https://doi.org/10.4321/S113976322015000300002

Cuetos, F., Suárez-Coalla, P., Molina, M. I. y Llenderrozas, M. C. (2015). Test para la detección temprana de las dificultades en el aprendizaje de la lectura y escritura. Revista Pediatría Atención Primaria, 17(66), 99-107.

Defior, S., Serrano, F. y Gutiérrez-Palma, N. (2015). Dificultades específicas de aprendizaje. Madrid: Síntesis.

Duk, C. y Murillo, F. J. (2016). La inclusión como dilema. Revista Latinoamericana de Educación Inclusiva, 1O(1), 11-14. https://doi.org/10.4067/SO718-73782016000100001

Echegaray, J. y Soriano, M. (2016). Conocimientos de los maestros acerca de la dislexia del desarrollo: Implicaciones educativas. Aula Abierta, 44, 63-69. https://doi.org/10.1016/j.aula.2016.01.001

Echeita, G. (2013). Inclusión y exclusión educativa. De nuevo, “voz y quebranto”. REICE. Revista Iberoamericana sobre Calidad, Eficacia y Cambio en Educación, 11(2), 99-118.

Echeita, G. (2017). Educación inclusiva. Sonrisas y lágrimas. Revista Aula Abierta, 46, 17-24. https://doi.org/10.17811/rifie.46.2.2017.17-24 
Echeita, G. y Domínguez, A. B. (2011). Educación inclusiva. Argumento, caminos y encrucijadas. Aula. Revista de Pedagogía de la Universidad de Salamanca, 17, 23-35.

Forteza, D. y Moreno, F. (2017). Procesos que obstaculizan la inclusión en la educación secundaria obligatoria. Muchas sombras y todavía pocas luces. Revista Aula Abierta, 46, 41-48. https://doi.org/10.17811/rifie.46.2.2017.41-48

Freire, P. (2001). Pedagogía de la indignación. Madrid: Morata.

Gasparini, A. A. y Culén, A. L. (enero, 2012). Tablet PCs- an assistive technology for students with reading difficulties? Comunicación presentada en The Fifth International Conference on Advances in Computer-Human Interactions. Universidad de Valencia.

Hatcher, P. J., Hulme, C. y Snowling, M. J. (2004). Explicit phoneme training combined with phonic reading instruction helps young children at risk of reading failure. Journal of Child Psychology and Psychiatry, 45(2), 338-358. https://doi.org/10.1111/j.14697610.2004.00225.x

Jeyasekaran, J. M. (2015). Effectiveness of visual auditory kinesthetic tactile technique on reading level among children dyslexia at Helikx open school and learning centre, Salem. International Journal of Medical Science and Public Health, 4(3), 315-318. https://doi.org/10.5455/ijmsph.2015.0511201467

López, M. (2012). La escuela inclusiva: una oportunidad para humanizarnos. Revista Interuniversitaria de Formación del Profesorado, 74(2), 131-160.

Murillo, F. J. y Hernández-Castilla, R. (2014). Liderando escuelas justas para la justicia social. Revista Internacional de Educación para la Justicia Social, 3(2), 13-32.

Papanicolaou, A. C., Simos, P. G., Breier, J. I. y Fletcher, J. M., Foorman, B. R., Francis, D., ... y Davis, R. N. (2003). Brain mechanisms for reading in children with and without dyslexia: A review of studies of normal development and plasticity. Developmental Neuropsychology, 24(3), 593-612. https://doi.org/10.1207/S15326942DN242\&3_05

Parody, L. M., Santos, M., Alcalá del Olmo, M. J. e Isequilla, E. (2019). El desafío educativo del siglo XXI: Relevancia de la cooperación entre familia y escuela. Espiral. Cuadernos del Profesorado, 12(24), 19-29. https://doi.org/10.25115/ecp.v12i24.2284

Pennac, D. (2008). Mal de escuela. Barcelona: Mondadori.

Porter, G. L. y Towell, D. (2017). Promoviendo la educación inclusiva. Claves para el cambio transformacional en los sistemas de educación. Recuperado de https://www.centreforwelfarereform.org/library/promoviendo-la-educacininclusiva.html

Pozo, J. I. (1999). Aprendices y maestros: La nueva cultura del aprendizaje. Madrid: Alianza.

Pujolàs, P. (2015). Marques de foc. Lliçons de pedagogia. Vic: Servei de Publicacions de la UVic.

Robledo, P. y García, J. (2014). Contexto familiar del alumnado con dificultades de aprendizaje o TDAH, percepciones de padres e hijos. Estudios sobre Educación, 26, 149-173. https://doi.org/10.15581/004.26.149-173

Slee, R. (2012). La escuela extraordinaria. Exclusión, escolarización y educación inclusiva. Madrid: Morata.

Soliman, M. S. y Al-Madani, F. M. (2017). The effects of multisensory-based instruction combined with brain-compatible environment techniques on reading fluency and reading comprehension of fourth-grade students with dyslexia. Croatian Journal of Education, 19(2), 363-397. https://doi.org/10.15516/cje.v 19i2.2190 
Soriano-Ferrer, M. y Piedra, E. (2017). Una revisión de las bases neurobiológicas de la dislexia en población adulta. Neurología, 32(1), 50-57. https://doi.org/10.1016/j.nrl.2014.08.003

Van Manen, M. (2003). Investigación educativa y experiencia vivida. Ciencia humana para una pedagogía de la acción y la sensibilidad. Barcelona: Idea Books.

Zuppardo, L., Serrano, F. y Pirrone, C. (2017). Delimitando el perfil emotivo-conductual en niños y adolescentes con dislexia. Reto XXI-Discapacidad y Educación, 1(1), 88-104.

\section{Breve CV de las autoras}

\section{Dolors Forteza}

Licenciada en Pedagogía y Doctora en Psicopedagogía por la UIB, en la que es profesora desde 1990. Codirectora del Máster oficial interuniversitario en Educación Inclusiva. Miembro del Grupo de Investigación de Escuela Inclusiva y Diversidad (GREID) y colaboradora en el Grupo de Investigación de Salud Global y Desarrollo Humano Sostenible (SG_DHS). Ha participado en diversas investigaciones y es autora de artículos sobre la atención a la diversidad. Su participación en congresos naciones e internacionales es notable. Directora de la Oficina de apoyo y responsable de las adaptaciones de acceso y admisión. Su línea de investigación principal es la Educación Inclusiva desde la etapa de infantil hasta la universidad. Ha ocupado distintos cargos de gestión, decana de la Facultad de Educación y vicerrectora de Docencia. ORCID ID: https://orcid.org/0000-00022053-9770. Email: dolorsforteza@uib.es

\section{Laura Fuster Coll}

Graduada en Educación Primaria por la Universidad de las Islas Baleares, con mención en Educación Musical y Artística (2017). Ha realizado el Máster oficial en Educación Inclusiva por la misma universidad (2018). Ha participado en un proyecto de Cruz Roja Española cuyo objetivo era contribuir al incremento del éxito escolar de niños y niñas de 6 a 16 años en dificultad social, incidiendo en los factores personales y sociales que lo favorecen. Ha asistido a diversos cursos y jornadas relacionadas con la mejora de la escuela y metodologías activas en el aula. Es voluntaria de la Asociación Dislexia y Familia (DISFAM). En la actualidad ejerce como maestra interina. ORCID ID: https://orcid.org/0000-0002-2135-8832. Email: laura.f.95@live.com

\section{Francisca Moreno-Tallón}

Diplomada en Educación Musical (2003), Licenciada en Psicopedagogía (2012) y Doctora en Educación Inclusiva y atención socioeducativa a lo largo del ciclo vital (2012) por la Universidad de las Islas Baleares. Ha realizado estancias en la Universidad de Salamanca, la Universidad de Oviedo y Reykjavík University de Islandia. Como profesora asociada ha impartido docencia en los estudios de Psicopedagogía y actualmente en los Grados de Educación Infantil y Primaria; también ha sido docente de cursos de postgrado de la UIB y de formación permanente en los Centros de Profesorado. Pertenece al Grupo de Investigación de Escuela Inclusiva y Diversidad (GREID). Autora de diversos artículos y comunicaciones en congresos. Sus líneas de investigación son: educación inclusiva y metodologías activas. ORCID ID: https://orcid.org/0000-0003-2923-4911. Email: francisca.moreno@uib.es 